\title{
Formal Methodology
}

\author{
A. Mutanen
}

\begin{abstract}
In general terms, methodology is a study of the entire scientific inquiry process: how science arrives at the posited goal. There are different kinds of goals for scientific inquiry. For example, goals may be epistemic (truth), aesthetic (simplicity) or several kinds of pragmatic goals (efficiency, economy, and explanatory power). It is not the concern of methodology what this goal happens to be. More generally, formal methods turned out to be effective tools in philosophical analysis, in the paper we will show this introducing the interrogative model and some basic properties of it, let us mention the covering law theorem. Finally we will formulate some philosophical implications of the model introduced.
\end{abstract}

Key words: logic, interrogative model, levels of methodological problems.

\section{Introduction}

The notion of formal methodology refers to logic or, rather, to philosophical logic. However, the scope and the aim of philosophical logic are not clear enough. It is reasonable to ask the question about the scope (and about the aim) of philosophical logic. Hintikka ([9]) takes quite a critical attitude to the whole approach: "Is there such a thing as 'philosophical logic'? Basically, my answer is 'no' " $([9], 1)$. The very idea behind Hintikka's negative attitude seems to be that there is just logic. Philosophers should do much work together with the logic and take a look at "which are of greatest interest and relevance to a philosopher's pursuits" ([9], 2). So, there is only logic, but philosophers have a philosophical interest and - in a sense logic is a tool, not a goal to a philosopher. In the same spirit we can say, that there is just methodology. Logical - formal - tools can, and should, be used if they have some philosophical relevance, that is, if the logic does some real (philosophical and conceptual) function. There is no reason only to formalize something that has been said before the formalization. According to Quine, the problem 
of translation has to be taken seriously: Why just try to translate? Is it possible just to translate?

In formal methodology the focus is not in formalization, but in methodology. Moreover, formal methodology need not be formalized. That is, the qualifier 'formal' does not refer to formalized. Here, we have the content of the distinction between philosophical logic and mathematical logic. This division is part and parcel of university schedule, for example, in Moscow and in Helsinki. In mathematical logic the intention is to study the formal structures and their properties; the intention is formal. In philosophical logic the intention is philosophical; the idea is to study philosophical notions. In this sense, formal methodology is a notion of philosophical logic.

In philosophy, or, in a general sense, in philosophical logic, formal - logical and mathematical - methods are used and studied very extensively. However, the systematic study of the nature and use of formal methods in philosophy has not been focal. The methodical role of formalization and the relationship between formallogical and philosophic-conceptual concept formation has not been fully characterized. Of course, quite a lot of work has been done, by, for example, Smirnov in Moscow. What is the real content of the assumption that formalism has to perform some philosophical task?

To get a better grasp of what is going on in formal methodology we may take a look at scientific inquiry processes. In scientific inquiry there is cooperation between different actors. The cooperative partners may have very different kinds of background. Some of the cooperators may have a practical background without any scientific experience (this may be the case also in scientific inquiry); moreover, the partners may, and usually have, experience in different fields of science. In practical life, each organization has its own routine ways to characterize and solve problems. Each field of science has its own tradition. How can cooperation overriding traditions be possible? How to do fruitful cooperation? For a philosopher the situation looks very challenging. How to conceptualize such cooperation? Moreover, a philosopher may be one of the cooperators. What can a philosopher give to such cooperation? What can a philosopher get from such cooperation? This enforces the philosopher to rethink the basic ideas - methodological basic questions. Formal methodology may help us to understand such a situation. 
Science is not done in a vacuum: the notion of tradition captures some essential aspects of the scientific inquiry process. The notion of tradition is not easy to characterize. The notion refers to a mental, social and practical level. At individual level tradition the notion of habitus captures some aspects of the notion. Habitus is something that can be seen from the behaviour of an individual. It is something personal but at the same something objective; something internal that the other can see. At the organizational level customs correspond to the habitus at an individual level. Customs refers basically to practical organizational behaviour. Besides, at the same it, refers to intellectual behaviour, to ways to identify interesting problems, for example. Science is both local and global: within a university department there is a tradition of its own, but at the same it has to be a member of global scientific community. (Cf. Merton's criterions.)

For example at the Department of Philosophy in University of Helsinki there is a long tradition of the use of formal methods in philosophical research. Several research groups study and use formal methods in their research work. To understand the philosophical environment at the department, let us mention names like: Eino Kaila, G H von Wright, Jaakko Hintikka, Oiva Ketonen, Erik Stenius, Veikko Rantala, Ingmar Pörn, Ilkka Niiniluoto, and Gabriel Sandu; all have worked at the department. All this has built a philosophical tradition which is still strong at the department. This tradition makes the choice of the methodological orientation natural or selfevident:

"The subject of formal methods in philosophy is intriguing but also for me at least puzzling, in more than one way. One puzzle is not unlike the predicament of the character in Moliere who is surprised to hear that he had been speaking French prose all his life long. What else could he have done? What other methods should - or could - I have possibly used in the philosophical work I have done?"([13])

Hintikka states that for him, the tradition of formal methods formulates a natural framework to do philosophical research. However, as Hintikka says, such a natural framework may be so selfevident that one does not react to it at all. It is something that one just does: what else could he (or I) do? Of course, Hintikka has made a conscious methodological choice. He has studied the very nature of methodology profoundly in his publications. He has 
both opened new paths for philosophical study and reflected on the philosophical importance of different methodic choices. But in general the problem is real: how have the methodological decisions been made in different fields of science?

The philosophical environment plays a central role in the focus of philosophical orientation. The philosophical environment shows how to do philosophy and how to identify important problems. In Helsinki the logic tradition has been strong and fruitful. All the students and researchers have a deep connection to the tradition. To have a connection, to work within and to reflect on a tradition are three different things. It is far from clear how to reflect on philosophical orientation. To reflect means that one has to look at the tradition (from some point of view). Reflection can neither be reduced into the analysis and classification of the actual practice nor into the analysis of ideal practice. The reflection captures some central aspects of the tradition and analyses the interdependencies between the factors analysed. So, reflection is something that Hintikka calls an explanatory model approach ([8]).

Let us take a step towards the reflective attitude to formal orientation. There is a proper need to understand the relationship between the different fields of science better. Sometimes philosophy - especially formal philosophy - is seen as a bridge builder between the different fields. In fact, [24] characterizes the spirit in Helsinki just in this sense:

In Helsinki " [t]here was no sign of Snow's two cultures in the intellectual climate of the department of philosophy of the University of Helsinki in the 70s. Hintikka lectured and supervised students from mathematics, philosophy and linguistics departments. It was another piece of common knowledge that at the doctoral level there are no disciplinary barriers: one just sees to it that he or she acquires the proper education in all the departments where the topic of the dissertation might lead her or him to" ([24]).

This demonstrates clearly the possibility to override the borders between different fields of science. However, to override, one just cannot ignore the borders, he or she has to be able to explicate notions and problems that have a fundamental methodological role. This shows a general meaning of the formal methodology we are searching for here. Overriding the borders supposes a very general methodological approach. The generality refers to abstractness - 
this abstractness is closely connected to formality. In fact, Hintikka's example demonstrates that such a methodological orientation can be done.

To achieve such a position, one has to reflect the very foundation of the scientific approach. However, in the tradition of formal philosophy reflection of the methods has not been in a central role. In fact, a formal approach may emphasize the practical aspects of the approach that is expressed by methodological motto taken from Nike 'Just do it' ([5]). Of course, in a practical work, like scientific inquiry, one has to emphasize the actual work in the Nike-spirit. However, there is still actual need for methodological discussion:

"The book is motivated by our curiosity but also by our discontent. Neither of us is content with the prominent histories of analytic philosophy currently on the market and we both believe that the discussion of general methodology of philosophy is in a pretty poor state. One of the most significant faults we see with such recent work is its failure to recognize and tackle the central place of formal methods. Shopworn narratives about the failures of logical positivism, the decline of formal methods in philosophy and the rise of intuitionsbased conceptual analysis, are neither entirely true nor particularly helpful. In any case, such talk has been overwhelmed by the ongoing buzz of interesting work from philosophers who look much more like Russell and Carnap than Rorty... . we can help to begin a fruitful conversation about the deep and interesting methodological problems that formal work in philosophy presents" ([7]).

To be fruitful it has to be discussion about something. That is, reflection has to have a specific content that encourages the search for fundamentals. So, the focus of the discussion has to be located somehow. [7] do this by focusing the discussion to epistemology:

"In the spring of 2005 we had the opportunity to work collaboratively on problems related to the application of epistemic logic and elements from formal learning theory to traditional epistemological questions. Given the nature of this topic, our conversations regularly turned to the more general question of the relationship between formal methods and philosophical investigation. We realized that some of the philosophers we most admire had never explicitly articulated their views on these questions and it occurred to us that it might be worth asking them" ([7]).

\section{Logic}

While speaking about formal methodology or formal philosophy it is not possible to avoid the discussion about logic and formal 
languages. However, we have to emphasize that formal methodology searched need not be formalized, and, moreover, formalized philosophical analysis need not be formal in a sense we intend. The use of logic and formal languages are explained to make it possible to explicate the argumentation:

"Formalization of an argument makes explicit its tacit assumptions. In some cases, one or more of these are clearly false, and one sees immediately what is wrong with the argument. In other cases, one is forced to think through the premises of the argument, and one may discover that was generally taken for granted may not be true" ([4]).

According to Føllesdal, formalization makes explicit tacit assumptions behind the argumentation. To do this the formalization focuses on some of the aspects in the argumentation, but at the same it hides something else. That is, by taking something explicitly under study, all the other things will be hidden. Of course, this is nothing new; if one observes one thing he or she does not observe something else. However, the focus of attention is a strategic choice. This opens the way to the critical evaluation of the situation. In a philosophical analysis, the use of formal methods has usually been restricted to formal or logical analysis of existing arguments or to the analysis of language of mathematics or of science. We should do a strategic evaluation of this choice ([11]).

Obviously "[o]ne of the most important cognitive abilities of people (and perhaps some other species) is the ability to reason" ([23], 5). In logic, the study of reasoning has been the formal study of argumentation, that is, argumentation theory. In the argumentation theory the emphasis has been in the study of relationships between premises and conclusion. The intention has not been in the characterization of actual argumentation but in the characterization of rules for rational or ideal argumentation. As Priest says "[l]ogic is the study of reasoning. It is not the study of how people actually reason. All too evidently, people often make mistakes when they reason. More importantly, the literature in cognitive psychology shows that people's reasoning ability appears to make systematic and predictable mistakes. Rather, logic is the theorisation of the norms of correct reasoning" ([23],5).

The very idea is clear: logic is a study of rational reasoning. However, this is not a reasoning that takes place in everyday 
argumentation between humans. It can be seen as a certain kind of ideal limit of human argumentation. So, logical meta-results give characterization theorems (maximality and minimality results) of human argumentation. A central feature of logic, which at the same makes it an effective tool in the argumentation theory, is that it is truth preserving: from true premises follow only true conclusions. If there is a false consequence then some of the premises have to be false. This is a foundation of hypotetico-deductive model of scientific inquiry. The traditional logical approach does not capture the dynamic aspects of actual human reasoning. In fact, one central aspect of hypotetico-deductive model is that it not dynamic but static.

However, logic is not merely a play with symbols but also epistemologically valuable. For example, Frege emphasized this aspect: "By insisting that the chains of inference do not have any gaps we succeed in bringing to light every axiom, assumption, hypothesis or whatever else you want to call it on which a proof rests; in this way we obtain a basis for judging the epistemological nature of the theorem." (Grundgesetze der Arithmetik, 1893) Similar emphasis can be found in the texts of Gödel and Turing. This emphasis is not necessarily connected to psychologism in logic. (See [19]) The attitude has been developed into a systematic approach in Hintikka's interrogative model of inquiry.

Unfortunately, as Priest denotes, humans do not follow the logical rules in actual reasoning. In fact, scientific argumentation does not follow the rules of logic either. This enforces us to search for better models of reasoning. The first model of reasoning to capture a philosophers' attention was the Socratic method of questioning or elenchus that Plato systematized into a practice of questioning games used in the Academy. In Topica Aristotle further developed a theory of interrogative argumentation. However, in the antique world the basic intention behind theories of interrogation games was pedagogic: to train students in philosophical and scientific reasoning $([12,16])$. Hintikka has developed a model of scientific reasoning that is "a framework for spelling out, explaining, and practicing good reasoning" ([15], 29). The model focuses especially on strategic aspects of inquiry process. The model is called interrogative model of inquiry. 
To get a better grasp of what is going on in the interrogative model let us to consider Aristotle's ideas for a moment. He focused his attention on the logical nature of answers in questioning games. Some of the answers were necessitated by earlier ones. In modern terms, these answers were logically implied by the earlier answers. The group has a very special nature of its own. In fact, Aristotle's study of this group of answers became the first study of logic in history. This branch has developed as an autonomous field of study deductive logic. Unfortunately the roots of the branch have been forgotten. There is also a set of answers that are not necessitated by earlier ones. This set of answers plays a special role in reasoning. In the interrogative model the idea is to give a strategic characterization of the information processing in reasoning ([16]).

\section{Knowledge}

The classical tripartite definition characterizes knowledge as welljustified, true belief. The purpose of definition is to define the meaning of the usual knowledge statement of the form ' $A$ knows that $p$ '. Moreover, the definition captures the so-called propositional knowledge.

To get a step further let us consider the notion of information. Let us say that $S$ is a sentence (in a language $L$ ). The class of models of $L$ determines a class of possibilities. The sentence $S$ divides the class of models of $L$ into two parts: into models in which $S$ is true and into models in which $S$ is false. This division is complete in the sense that each model makes the sentence true or false but not both. The sentence $S$ excludes the class of model in which it is false in an obvious way. The semantical information of $S$ is determined on the basis of this fact $([1,14])$.

In the same spirit, if an agent knows that $p$ it means that the agent is entitled to behave as if $\mathrm{p}$ would be true. More generally the knowledge excludes some of the possible courses of events. The possible courses of events are divided into the two groups according whether the knowledge excludes it or not. However, this analysis connects knowledge to the notion of information and to the notion of action. This notion is natural in the framework of interrogative model of inquiry, but the traditional tripartite definition does not capture it. We will consider this a little bit closer later. 


\section{Interrogative model of inquiry}

Interrogative model of inquiry is a general framework developed by Hintikka and his associates in the 1990's. The fundamental idea behind the interrogative model is to consider questioning and answering systematically as a method of knowledge seeking. A fundamental part of the model is the logic of questions and answers. However, questioning and answering is not abstract logical work but actual search for new knowledge. That implies that without epistemic notions (especially modern epistemic logic) one cannot formulate the model adequately ([16]).

In the simplest case the model is characterized by the following features: (i) There is only one oracle. (ii) The set of answers the oracle will provide remains constant throughout the inquiry. (iii) All of the oracle's answers are true, and known by the inquirer to be true. This simple case allows us to formulate several interesting general meta-results of the interrogative model. Moreover, there are several theoretically and practically interesting aspects of the model that can be seen from the simple formulation.

The condition (i) does not cause any principal restrictions. By suitable coding the model canbe applied to any case in which the number of oracles is enumerable. The condition (ii) does not give an adequate picture of all the cases. Innovation processes are practical example of the context in which actor can change the set of possible future answers. It is possible to formulate a model in which inquirer's action (or some other practical change in the environment) chances the set of available answers. For example the set of observable things depends on the observer's actions. (See [17]) The condition (iii) is not true in every case. There are lots of interesting practical applications in which we would like to relieve the condition. To do this we need one extra operator into the interrogative model. The operator is called bracketing operator which shows that the information given by the oracle is not certain. (See [16]) However, the simple model is worth of closer study. We can formulate some interesting results by using this simple model. It is possible to generalize these results to hold also in the generalized model ([16]).

In basic formulation of the interrogative model the argumentation is relative to a first-order language. The oracle gives truthful answers - the answers are truths about a particular model $\mathbb{M}$ of the 
given language. An interrogative argumentation proceeds relative to the model. Let $C$ be a sentence of the language, and let $T$ be a set of sentences of the language. We call $T$ as the background knowledge of the inquirer. By simplicity we assume that $\mathrm{T}$ is true in the model. The inquirer is trying to derive $C$ from $T$ together with some auxiliary information about the model. When $C$ is derivable from $T$ in $\mathbb{M}$, we say that $\mathrm{C}$ is a model consequence of $T$ in $\mathbb{M}$, and express this by

(1) $\mathbb{M}: T \vdash C$.

Clearly, if no questions are allowed, $\mathbb{M}$ becomes irrelevant and the relation of model consequence reduces to the usual relation of deductive consequence. Conversely, if there is no restriction in the questions asked and answers given by the oracle, the model consequence reduces to the notion of truth in the model $\mathbb{M}$. Already this shows the theoretical interest of the interrogative model. For simplicity we say that $C$ is interrogatively inferred from $T$ in $\mathbb{M}$ if (1) holds. So, the interrogative model studies interrogative reasoning ([11]).

All the additional information brought into the argument comes from an outer source of information. These are understood as answers to questions asked by the inquirer. There are no principal restrictions to the questions asked. This open possibility for additional information makes the argumentation dynamic and open-ended. In practice, the additional information comes via observations, experiments or some other kinds of sources. In such cases the additional information can be called empirical.

The interplay between logical and empirical information in the rational argumentation is of certain importance. Because of theoretical reasons they cannot be separated effectively in practice. The practical separation supposes that increase of empirical information and explication of the existing information would be effectively separated. However, it imposes extreme logical complexity to the reasoning to do this. This fact throws some dark clouds to traditional decision theories $([11,14])$.

To proceed on, let us inspect some properties of the model that show more obviously the newness of the model. Let us assume that the set $\Phi$ includes all the possible answers that the arguer may in principle get during the argumentation process. With help of the set $\Phi$ it is possible to close the process. It is possible to prove 
the following theorem, which shows that the open-ended and closed models are factually equivalent:

THEOREM 1 (Completeness Theorem). If $\Phi$ is the set of all available answers and $T$ the set of initial premises, then a conclusion $C$ can be established in a model $\mathbb{M}$ by means of interrogative logic if and only if $(\Phi \cup T) \vdash C$.

The theorem shows that the open-endedness does not cause logical or theoretical problems. However, it does not annihilate the essential difference between the two approaches. The notion of model completeness studied by Abraham Robinson 1953 shows this. Let $T$ be a consistent theory expressed in a first-order language $L$, and let $\mathbb{M}$ be a model of $T$, i.e., $\mathbb{M} \models T$. The diagram $D(\mathbb{M})$ is the set of atomic and negated atomic sentences of the extended language $L(M)$, where $M$ is the domain of the model $\mathbb{M}$, such that for all $S \in D(\mathbb{M}): \mathbb{M} \models S$. We say that $T$ is a model complete if for all $S \in L(M)$, i.e., the sentences may contain additional names for individuals, either $T \cup D(\mathbb{M}) \vdash S$ or $T \cup D(\mathbb{M}) \vdash \neg S$. So, the completeness theorem shows the close connection of the interrogative model to the model theoretical logic á la Abraham Robinson. This has several deep logical and philosophical implications ([10]).

The following theorem is of crucial philosophical importance. The analysis of the theorem shows several central ideas behind the interrogative model. The theorem is proved in [16].

THEOREM 2 (Extended Interpolation Theorem). Assume that $T(P)$ is a consistent theory such that $C$ can be interrogatively derived from the theory $T(P)$ in a model $\mathbb{M}$, i.e., $\mathbb{M}: T(P) \vdash C$; and not:T $(P) \vdash C$. Then there is an interpolant $I\left(a_{1}, \ldots, a_{n}\right)$ such that each non-logical constant of $I\left(a_{1}, \ldots, a_{n}\right)$ occurs in both $T$ and $C$ except for a finite number of individual constants $a_{1}, \ldots, a_{n}$. (i) $\mathbb{M}$ : $T(P) \vdash I(a 1, \ldots$, an $)$, (ii) $I(a 1, \ldots$, an $) \vdash C$.

The theorem shows the close interconnection of the interrogative model to the traditional logical (deductive) model. However, there is a deep logical and philosophical difference between the models. The analysis of the theorem, and the proof of the theorem, shows several interesting philosophical facts. The individual constants that occur in the theorem are answers to inquirer's questions. The number of the constants shows the empirical depth of the theorem (proof). This corresponds to the surface information defined by Hintikka ([9]). 
However, if we compare the proof of the theorem and the corresponding theorem in logic we observe that the structures of the proofs are similar. Moreover, the questions correspond to existential instantiations in the logical proof. Fortunately it is possible to generalize the observation. The interrogative model shows that there is a close connection between deductive reasoning and general interrogation (empirical reasoning): from the strategic point of view they can be seen as two parallel processes. This can be formulated as a theorem - the Strategy Theorem ([16]).

The observation shows the importance of strategic aspects in a study of reasoning. Strategy theorem shows that the interrogative model is an effective method for the study. The strategy theorem opens a new path to the study of strategies of reasoning. The strategy theorem is a fundamental theorem of the interrogative model: the interrogative model is a strategic study of inquiry. This imposes that the interrogative model is a general approach to evaluate the research processes ([14]).

Before we will go on, let us formulate the following covering law theorem that generalizes the extended interpolation theorem. The theorem has a corresponding theorem in logic. However, the following theorem has a special task in the philosophy of science. It is a key theorem which opens new light into the theory of explanation. The theorem shows that the interrogative model can be developed as a proper formalized theory, but at the same the theory has a deep philosophical content. The theorem is proved [16].

THEOREM 3 Covering law Theorem: Let $T$ be a theory in the first-order language $L$ and $F=F\left[b_{1}, \ldots, b_{n}\right]$ be a sentence such that $F\left[x_{1}, \ldots, x_{n}\right]$ is a formula of $L$ and $b 1, \ldots, b n \in M$. Let $\mathbb{M}: T \vdash F$ be established by means of a constant set of answers $A$. Let us assume that $A$ is consistent and that $T$ does not entail $F\left[b_{1}, \ldots, b_{n}\right]$. If other constants than $b_{1}, \ldots, b_{n}$ of $F$ do not occur in $A$ and if $b_{1}, \ldots, b_{n}$ do not occur in $T$, then there is a formula $H\left[x_{1}, \ldots, x_{n}\right]$ of $L$ such that (i) $T \vdash(x 1) \ldots(x n)(H[x 1, \ldots, x n] \rightarrow F[x 1, \ldots, x n])$; (ii) $A \vdash H[a 1, \ldots, a n]$, none of the constants of $F\left[x_{1}, \ldots, x_{n}\right]$ occur in $H\left[x_{1}, \ldots, x_{n}\right]$. 


\section{Interrogation}

In the interrogative reasoning there are two kinds of steps: logical steps and interrogative steps. Logical steps are as usual truth preserving. Interrogative steps search new information into the argument. Answers are used in the reasoning as any other item of information. However, if the inquirer does not know where the new information comes from we are not dealing with rational reasoning but with mere guesswork. The information coming from the oracle is new in the logical sense of the word: the information cannot be deduced from the information the arguer has at the moment. The inquirer has to receive the information as a response to an earlier question asked from the oracle ([16]).

In general there is no guarantee that the information would be true. However, the structure of interrogative reasoning shows that if all the forthcoming information is true (and also known to be true) then the resulting conclusions will also be true (and known to be true). In this case we will get a logic of discovery in a strict logical sense. Moreover, if the answers are known to be true then the inquirer get new knowledge as a result of the inquiry. In this case the method used is reliable in a strict logical sense.

However, if it may happen that some of the answers would not be true, the situation will be changed. In this case, the inquirer has to evaluate the forthcoming information and some of the forthcoming information is reasonable to be marked uncertain. All the consequences that depend on this item of information also have to be marked uncertain. Of course, some uncertain item of information may become certain if some further information supports it. To handle this situation we have a rule of bracketing and unbracketing ([16]).

However, in this case we will not have any more a logic of discovery. The idea is that the inquirer searches for knowledge in uncertainty. The inquirer has to have a strategic approach to the process. He or she has all the time to strategically evaluate all the steps. Hence, we have a logic of justification. The bracketing and unbracketing rules show the interconnection between discovery and justification. There is a close strategic link between the two. However, we can see that the logic of justification is strategically more complex than the mere logic of discovery ([14]). 
In the above discussion we have discussed the questions in an interrogative process in one specific sense, namely request of additional information. In a sense, this is a very central meaning in the interrogative model. Inquiry is a goal-directed process. The final goal can be expressed via questions. However, the structure of the interrogative model separates the two types of questions. The questions that express the final goal are called principal questions and questions that request for additional information are called operational questions.

The inquirer consults one source of information or another. The role of the information is to add new information into the inquiry process. The information directs the inquiry process in an obvious way. However, this implies that the result of inquiry is dependent on the source of information. The inquiry process may end to this or to that result depending on the source of information used. This source dependency is a structural property of the inquiry process. That is, it is a strategic principle that is built up to the structure of the interrogative model ([14]).

Our discussion has been discussion about information, not about knowledge. The reason has been built into the structure of interrogative model. The model does not presuppose that the inquirer knows or believes the forthcoming information. In some special cases the inquirer may even doubt the truth of the information. However, all the time the discussion has been about the information achieved during the process. Moreover, the end product of the inquiry may still be mere information, that is, there are no general guarantees that the inquiry process would end up to knowledge. It is possible to study the structure of inquiry processes and find out whether the process is or is not reliable. This is a strategic problem of the whole interrogative model ([19]).

\section{Formal methodology}

In the interrogative model strategic aspects of the questioning process are emphasized. However, it is possible to characterize them from a different point of view. By classifying different kinds of questioning settings we can at the same give different kinds of methodological problems. They correspond to Kelly's classification of methodological problems. 
Usually, in methodology textbooks of empirical inquiry there is a chapter that considers reliability and validity of the inquiry. Clearly, this is a problem of methodological relevance. However, the basic idea is to discuss the reliability of this specific inquiry (in a specific context) only. Usually, the discussion is solely of rhetorical value. There is no theoretical or methodological discussion about the general setting of the inquiry. The language is purely descriptive. The reason for this is that the structure of the research problem discussed is fixed in an obvious way. This gives us the lowest level of methodological problems according to [17]. At this level methods are determined by a theory, that is, questions and the questioning strategy is determined by the experimental setting. At this level the questioning strategies are closed. The methodological discussion just shows that the required standards are fulfilled.

To bring about a methodologically more interesting discussion there have to be some factors that can behave as variables. For example, in statistics there are results - for example, the law of large numbers - that show that a certain method gives a reliable result for a certain kind of evidence. This implies that the inquirer has to justify the types of operational questions he or she uses during the inquiry process. The type of operational questions determines the sequences of additional information in an obvious way. Such argumentation gives general methodological information about the inquiry strategy and hence also about the reliability of the inquiry. This is Kelly's second level of methodological problems. At this level we have results that can be applied in several different fields of science.

In philosophy of science the question of the scope of a given $\operatorname{method}(\mathrm{s})$ plays, or should play, a central role. For example, the study of the relationship between quantitative and qualitative inquiry can be seen as an example of this kind of question. A problem encompasses the possible situations (problem settings) into which a method can (or cannot) be applied. According to the interrogative model this concerns the logic of principal questions of the interrogative model. This is Kelly's third level of methodological problems. At this level it is possible to distinguish different fields of science on a methodological basis. For example, von Wright ([25]) distinguished humanities and natural sciences in just this sense. 
It is possible to look at the scientific inquiry as a problem solving activity ([18] and [22]). However, it is not clear enough what this means: A scientist starts with a problem and searches for a solution. This opens some problems: How to solve it? Is the problem solvable with the method used? At this level, the inquirer may search, or compare, for methods to solve the problem. In the interrogative model the inquirer has - or should have - a questioning strategy (strategy theorem). This level is a study of questioning strategies. That specifies Kelly's fourth level of methodological problems.

However, it is possible to take one more step in our abstraction process. This can be done by considering the third and fourth levels together, which allows us to consider problem solving in general. This gives us Kelly's fifth level of methodological problems. Examples of problems of this level are, for example, the possibility of logic of discovery, logic of justification, and the theory of explanation. At this level interrogative model is a general theory of reasoning (different meta-theorems). At this level the general theory of interrogative processes is the general theory of problem solving ([14], [16], [3]).

The levels of methodological problems imply to a different kind of formality. Methodological problems of the lowest level are connected directly to practical problems in scientific inquiry. At that level it is not possible to formulate general methodological problems. Hence, we said that the problems an this level are mainly of rhetorical value. To have more interesting problems methodologically we have to make the problem setting more formal - less dependent on the specific context and specific topic. The hierarchy is built accordingly: at the fifth level there is not direct connection to any specialities of the specific local details. The fifth level formulates a general theory of problem solving - the formal methodology we were searching. Of course, this can be formalized by using a formal language. However, the formalization is neither needed nor supposed. So, in formal methodology formalism is neither a necessary nor sufficient condition ([14]).

Methodology should allow us to evaluate the rationality of the research process. To do this, the process should have both a descriptive and a normative component. It is connected to the existing scientific tradition, but cannot be just documentation of an 
actual inquiry. To evaluate it also has to be normative: it has to give some standards to the evaluation. In methodology - at least in formal methodology - it is possible to characterize the reliability of methods in different possible cases. As we have seen the interrogative model is an answer to the request for this kind of methodology ([14], [6], [17]).

In the classification notions like method, problem, solution etc. came up. These are central methodological notions that require explication. In the explication it is natural to use formal methods. In this task it is possible to use computational methods, like Kelly [17], Hendricks [6], Mutanen [19], or logical methods, like Hintikka, Halonen, and Mutanen [16], Hintikka [14]. Independent of which formal methods are used, the formal methods must do some philosophical work. That is, allow us to reflect the philosophical framework in which the problem takes place at a conceptual, theoretical, and methodological level. The formal methodology formulated above can be used as a framework in stimulating cooperation between different fields of sciences.

Formalism as such is not, or should not, be the final goal of formal methodology. Formal methods are tools that are actively used in the study. The final goal is to give a better understanding of the philosophically central notions and the interconnection of such notions. Formal methodology is still philosophy, and it is closely connected to other philosophical approaches ([7]).

\section{References}

[1] Bar-Hillel Y. and Carnap R. Semantic Information // British Journal for the Philosophy of Science 4, 1953. P. 147-157.

[2] Burgin M. Three aspects of Super-recursive Algorithms and Hypercomputation or Finding Black Swans // Theoretical Computer Science 317, 2004. P. 1-11.

[3] Engeström Y. Activity Theory and Individual and Social Transformation, in Engeström, Y., R. Miettinen, and R-L. Punamäki (eds.) // Perspectives on Activity Theory, Cambridge University Press, 1999.

[4] Føllesdal D. // Hendricks and Symons, 2005.

[5] Haack S. // Hendricks and Symons, 2005.

[6] Hendricks V. F.The Convergence of Scientific Knowledge - a View from the Limit // Kluwer Academic Publishers, 2001.

[7] Hendricks V. F. and Symons J. Formal Philosophy // Automatic Press, 2005.

[8] Hintikka J. Models for Modalities // D. Reidel Publishing Company, 1969.

[9] Hintikka J. Logic,Language-Games and Information : Kantian Themes in the Philosophy of Logic // Clarendon Press, 1973. 
[10] Hintikka J. The logic of science as a model-oriented logic. In P. Asquith and P. Kitcher (eds.) // PSA, vol. 1. East Lansing, Michigan: Philosophy of Science Association, 1984. P. 177-185.

[11] Hintikka J. What Is the Logic of Experimental Inquiry? // Synthese 74, 1988. P. 173-188.

[12] Hintikka J. The Principles of Mathematics Revisited / Cambridge University Press, 1996.

[13] Hintikka J. // Hendricks and Symons, 2005.

[14] Hintikka J. Socratic Epistemology // Cambridge University Press, 2007.

[15] Hintikka J. and Bachman J. What If...? Toward Excellence in Reasoning // Mayfield Publishing Company, 1991.

[16] Hintikka J., Halonen I. and Mutanen A. Interrogative Logic as a General Theory of Reasoning in R.H. Johnson and J. Woods (eds.) // Handbook of Practical Reasoning, Kluwer Academic, 2002.

[17] Kelly K. The Logic of Reliable Inquiry // Oxford University Press, 1996.

[18] Laudan L. Progress and its Problems: Toward a Theory of Scientific Growth // Berkeley University of Califormia Press, 1977.

[19] Mutanen A. From Computation to Truth via Learning // Helsinki, 2004.

[20] Mutanen A. Methodology of Engineering Science as a Combination of Epistemic, Ethical and Aesthetic Aspects, in Hyldgaard, Steen (ed.) // Philosophy in Engineering, Academica, 2007.

[21] Niiniluoto I. The Aim and Structure of Applied Research // Erkenntnis 38, 1993. P. 1-21.

[22] Niiniluoto I.Truthlikeness // D. Reidel, 1987.

[23] Priest G. Logic // The Newsletterfor Philosophical Logic and Its Applications, Springer, 2007.

[24] Sandu G. // Hendricks and Symons, 2005.

[25] von Wright G. H. Explanation and Understanding, Routledge and K. Paul, 1971. 